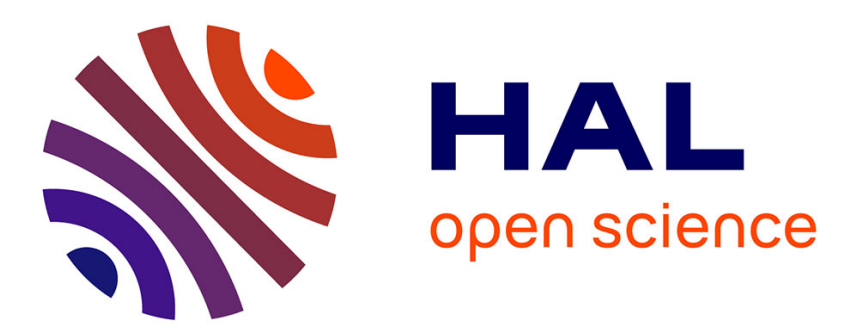

\title{
A single segment differential tube model with interchain tube pressure effect
}

\author{
Sunil Dhole, Adrien Leygue, Christian Bailly, Roland Keunings
}

\section{To cite this version:}

Sunil Dhole, Adrien Leygue, Christian Bailly, Roland Keunings. A single segment differential tube model with interchain tube pressure effect. Journal of Non-Newtonian Fluid Mechanics, 2009, 161 (1-3), pp.10-18. 10.1016/j.jnnfm.2009.03.014 . hal-01004946

\section{HAL Id: hal-01004946 \\ https://hal.science/hal-01004946}

Submitted on 14 Oct 2017

HAL is a multi-disciplinary open access archive for the deposit and dissemination of scientific research documents, whether they are published or not. The documents may come from teaching and research institutions in France or abroad, or from public or private research centers.
L'archive ouverte pluridisciplinaire HAL, est destinée au dépôt et à la diffusion de documents scientifiques de niveau recherche, publiés ou non, émanant des établissements d'enseignement et de recherche français ou étrangers, des laboratoires publics ou privés. 


\title{
A single segment differential tube model with interchain tube pressure effect
}

\author{
Sunil Dhole ${ }^{a}$, Adrien Leygue ${ }^{b}$, Christian Bailly ${ }^{\mathrm{a}, *}$, Roland Keunings $^{\mathrm{c}}$ \\ a Unité de Chimie et de Physique des Hauts Polyméres, Université Catholique de Louvain, B-1348 Louvain-la-Neuve, Belgium \\ ${ }^{\mathrm{b}}$ Department of Materials Science and Engineering, National Technical University of Athens 157 80, Greece \\ c CESAME, Université Catholique de Louvain, B-1348 Louvain-la-Neuve, Belgium
}

We develop a single segment differential tube model including interchain tube pressure effect (ITPE) [G. Marrucci, G. Ianniruberto, Interchain pressure effect in extensional flows of entangled polymers, Macromolecules 36 (2004) 3934-3942], able to describe the non-linear behaviour of entangled linear polymers. The model accounts for the effect of flow on the tube length and diameter. It is presented in two versions, depending on which tube dimension is assumed to deform affinely. The classical relaxation mechanisms, i.e., reptation, stretch dynamics, convective constraint release (CCR), as well as finite extensibility, are incorporated in a simple manner; hence the model allows an explicit comparison of the relative importance of various effects. A striking result is the insignificance of finite extensibility and the detrimental influence of CCR for moderately entangled systems when ITPE is taken into account. For highly entangled systems, CCR regains importance to avoid the well-known shear stress instability. The proposed model is able to make quantitative predictions of steady elongational and shear data for monodisperse melts, while transient values are less accurate but within experimental errors.

\author{
Keywords: \\ Tube model \\ Interchain tube pressure effect \\ Convective constraint release \\ Finite extensibility
}

\section{Introduction}

Doi and Edwards [2], following the concept of reptation by de Gennes [3], first proposed a tube model to describe the rheology of linear entangled polymers in the linear viscoelastic regime. Steady progress over two decades has made quantitative predictions possible for linear properties [4-6]. On the other hand, the development of theories for the non-linear response of entangled polymers is much more limited and even in the case of monodisperse linear chains, additional relaxation-mechanisms are still proposed today to improve the description of the flow properties [1]. The Doi and Edwards (DE) model [2] was a first attempt to build a tube-based constitutive equation for linear polymers. Though the predictions of the DE model are in very good agreement with experimental data for step deformations, it fails even in the prediction of qualitative non-linear features for other types of flow. In particular, the DE model predicts a constitutive instability in steady shear.

In an attempt to improve the theory, Marrucci and Grizzuti [7] and Marrucci [8] successfully pointed out stretch dynamics and convective constraint release (CCR) as key elements to overcome the DE model's limitations. Marrucci and Ianniruberto [9] proposed a promising single segment differential tube model that incorporates both chain stretch and CCR in a coupled representation. This

\footnotetext{
* Corresponding author. Tel.: +32 1047 3560; fax: +32 10451593 .

E-mail address: christian.bailly@uclouvain.be (C. Bailly).
}

model is simple enough to be easily used for complex flow simulations [10], but yet retains the necessary physics. Using the concept of CCR and stretch dynamics, Mead et al. [11] proposed an integral tube model, which can make quantitative predictions in fast shear flows. Another integral model, known as the molecular stress function model (MSF) is based on the assumption of a strain-dependent tube diameter and has been proposed by Wagner et al. [12]. Graham et al. [13] have developed a comprehensive differential tube model where the effects of reptation, chain stretch, and CCR are derived from a microscopic stochastic partial differential equation that describes the chain contour dynamics. This model can make quantitative predictions in linear and non-linear shear flows and slow elongational flows $\left(\dot{\varepsilon}>1 / \tau_{\mathrm{r}}\right.$, where $\tau_{\mathrm{r}}$ is the Rouse relaxation time). Likhtman and Graham [14] have simplified the Graham et al. [13] model to a single segment differential model, which is known as "Rolie-Poly", and makes qualitatively similar predictions to those of Graham et al. [13]. Recently, Leygue et al. [15] have proposed a new differential tube based constitutive model, which incorporates, in a full chain approach (multi-segment), the major molecular mechanisms thought to be important to describe the flow of entangled polymers: reptation, contour-length fluctuations, thermal and convective constraint release, chain stretch, and finite extensibility of the polymeric chains. The pertinent literature to date has been thoroughly reviewed by Dealy and Larson [16]

All the tube theories mentioned above predict elongational thickening around an elongation rate $(\dot{\varepsilon})$ of $1 / \tau_{\mathrm{r}}$ for linear polymer melts. However, the data of Bach et al. [17] on monodisperse 


\section{Nomenclature}

$a \quad$ tube diameter ( $\mathrm{m}$ )

$a_{0} \quad$ equilibrium tube diameter ( $\mathrm{m}$ )

B conformation tensor

C matrix square of tensor $\mathbf{B}$

E deformation tensor

$f \quad$ finite extensibility modification

$G_{\mathrm{N}}^{0} \quad$ plateau modulus (Pa)

$l \quad$ tube length $(\mathrm{m})$

$l_{0} \quad$ equilibrium tube length $(\mathrm{m})$

$N_{\mathrm{k}} \quad$ number of Kuhn steps (-)

$N_{1} \quad$ first normal shear stress difference (Pa)

$T_{12} \quad$ shear stress $(\mathrm{Pa})$

$t \quad$ time (s)

$\operatorname{Tr} \quad$ Trouton ratio $\left(\eta_{\mathrm{el}} / \eta_{0}\right)$

$u \quad$ orientation vector distributed on the unit sphere

$Z \quad$ number of entanglements

\section{Greek symbols}

$\alpha \quad$ normalized tube diameter $(-)$

$\beta \quad$ numerical CCR factor $(-)$

$\delta \quad$ unit tensor $(-)$

$\lambda \quad$ normalized tube length $(-)$

$\sigma \quad$ stress tensor $(\mathrm{Pa})$

$\dot{\gamma} \quad$ shear rate $\left(\mathrm{s}^{-1}\right)$

$\varepsilon \quad$ Hencky strain $(-)$

$\dot{\varepsilon} \quad$ elongation rate $\left(\mathrm{s}^{-1}\right)$

$\eta_{0} \quad$ zero shear viscosity (Pa s)

$\eta_{\mathrm{el}} \quad$ steady elongational viscosity (Pa s)

$\tau_{\mathrm{d}} \quad$ reptation time (s)

$\tau_{\mathrm{p}} \quad$ tube relaxation time (s)

$\tau_{\mathrm{r}} \quad$ Rouse relaxation time (s)

polystyrene instead show an elongational thinning behaviour $(-0.5$ power law) for $\dot{\varepsilon}>1 / \tau_{\mathrm{r}}$. This behaviour for polystyrene has later been confirmed by Luap et al. [18] from stress and birefringence experiments in elongational flows. Recent published data of Nielsen et al. [19] also show the same behaviour, again for polystyrene. The tube theory by Leygue et al. [15] can quantitatively predict this elongational thinning behaviour for polystyrene, but only for unrealistically low values of finite extensibility ( $b=12$, instead of $b=66$ the value derived from microstructure) [20]. In order to remedy this issue, Marrucci and Ianniruberto [1] have proposed to include an interchain tube pressure effect (ITPE), based on an original idea by Doi and Edwards [2]. This concept, which is based on tube diameter dynamics is able to predict the observed elongational thinning in a straightforward way and qualitatively explains the observed scaling of tensile stress with elongation rate. As a follow-up work, Marrucci and Ianniruberto [21] have developed a 2D constitutive model for ITPE, which can make qualitative predictions both in shear and elongational flows. More recently, Wagner et al. [22,23] have incorporated the idea of ITPE, along with the finite extensibility in their MSF model and successfully predicted the Bach et al. [17] and Luap et al. [18] data.

So, the state of the art on tube models is that, only the MSF model with ITPE [22] can predict the transient and steady elongational data for linear polymer melts at high elongation rates $\left(\dot{\varepsilon}>1 / \tau_{\mathrm{r}}\right)$. This model, however, requires the knowledge of the entire relaxation spectrum, and being an integral model, makes numerical simulations heavy. Therefore, the aim of the present work is to develop a simple, full tensorial single segment differential tube model, which can make (semi-)quantitative predictions in high shear and elongational flows. This model is based on all

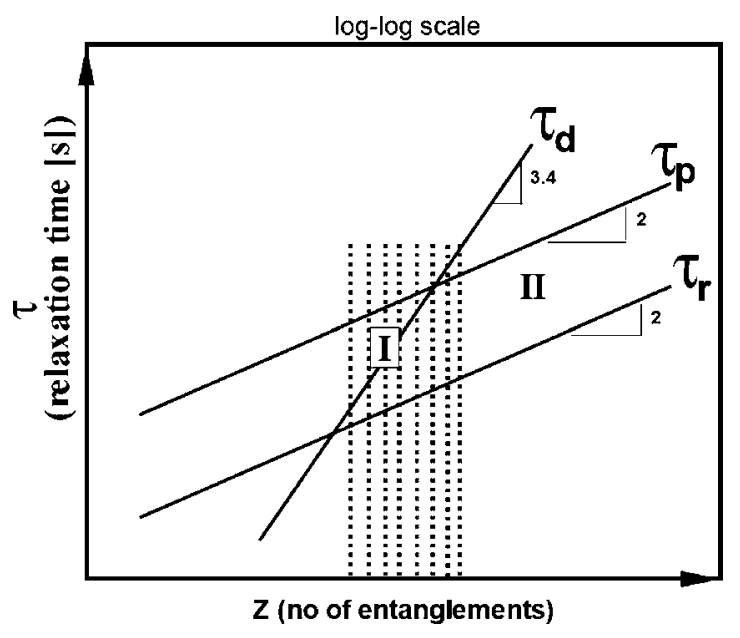

Fig. 1. Relaxation time $(\tau)$ vs. number of entanglements $(Z)$ : regimes for different $\tau_{\mathrm{d}} / \tau_{\mathrm{p}}$ ratios

the above mentioned relaxation mechanisms including ITPE. It also serves the purpose of studying the influence of different relaxation mechanisms in relation to each other. The proposed model hence includes reptation, stretch dynamics, finite extensibility, convective constraint release, and ITPE. Since it is a "single segment" model, thermal constraint release effects can only appear through a rescaling of the reptation time $\tau_{\mathrm{d}}$.

In order to include the concept of ITPE into the full tensorial model, tube diameter dynamics need to be converted into tube length dynamics. Thus, this paper starts with the section, "tube dynamics", dealing with this issue. Next, the development of the single segment model is presented. In the following section, the qualitative and quantitative performance of the model is discussed. In the last section, the main conclusions are exposed.

Before moving to the next section, we would like to clarify the concept of moderately and highly entangled systems. Fig. 1 presents a qualitative picture of the relation (on a log-log scale) between characteristic relaxation times $\left(\tau_{\mathrm{d}}, \tau_{\mathrm{r}}\right.$ and $\left.\tau_{\mathrm{p}}\right)$ and the number of entanglements $(Z)$. Classical tube models $[9,15]$ include two characteristic relaxation times, i.e., the Rouse time and the reptation time. The scaling of $\tau_{\mathrm{r}}$ and $\tau_{\mathrm{d}}$ vs. molar mass ( or $Z$ ) is well established. The characteristic time $\tau_{\mathrm{p}}$ is the tube relaxation time representing ITPE [1]. As shown by Marrucci and Ianniruberto [1], $\tau_{\mathrm{p}}$ is proportional to $M^{2}$ with an unknown pre-factor $(\sim 50)$. Therefore, qualitatively, Fig. 1 identifies two zones of linear entangled polymer systems. Zone I (shaded area in Fig. 1) is for moderately entangled systems $\left(\tau_{\mathrm{p}}>\approx \tau_{\mathrm{d}}\right)$, and Zone II for highly entangled systems $\left(\tau_{\mathrm{p}}<\tau_{\mathrm{d}}\right)$.

\section{Tube dynamics}

In this section, two different scalar models for tube dynamics, based on alternative deformation assumptions for a tube segment, are developed in the special case of an elongational flow. Later, in the next section, these scalar models are generalized to full tensorial models.

For a tube segment, characterized by length $l$ and diameter $a$ one can ask about the way it changes in an extensional flow, when the tube segment is already aligned with the flow. The first option is of course to consider that everything deforms affinely. Under this assumption, the volume $V=l a^{2}$ of the tube segment is preserved at all times.

However, the assumption of incompressibility of the material can not be used, as tubes of different chains certainly overlap and the tube does not have the characteristics of a solid object. If the tube does not deform affinely then one has to find how $l$ or $a$ evolve and 
identify the relation between them. Doi and Edwards [2] calculate the equilibrium length $l_{0}$ of the primitive path of a chain confined in a tube of a given diameter $a_{0}$. In this derivation, they first compute the entropy of such a confined chain and then derive the primitive path length that maximizes this entropy. They obtain the following scaling relationship:

$l_{0} \sim a_{0}^{-1}$.

Let us define the relative tube diameter $\alpha$ and relative tube length $\lambda$ as follows:

$\alpha=\frac{a}{a_{0}}$,

$\lambda=\frac{l}{l_{0}}$,

where $a_{0}$ and $l_{0}$ are the respective equilibrium values. Eq. (1) now reads:

$\alpha=\lambda^{-1}$.

Eq. (4) is strictly valid at equilibrium, but we assume the same scaling to hold under flow conditions. Indeed, the flow-induced perturbation of the tube diameter is a local phenomenon with a characteristic length of about the entanglement distance. The corresponding chain section explores the tube with a characteristic equilibration time around the segmental time $\left(\tau_{\mathrm{e}}\right)$. Hence the local tube diameter vs. length equilibration can be considered instantaneous at deformation rates up to and even above $1 / \tau_{\mathrm{r}}$ for significantly entangled chains. Thus we should be able to exploit Eq. (4) even under the fast flow conditions. As will be shown in following sections, the quantitative performance of the proposed model in the nonlinear regime supports the validity of the assumption. It is also worth mentioning that the MSF model with ITPE [22] uses the same scaling and can make quantitative predictions at deformation rates above $1 / \tau_{\mathrm{r}}$.

In their paper on ITPE [1], Marrucci and Ianniruberto do not really address the dynamics of the tube segments, since they focus on what they call "Zone 2" (Fig. 1 in [1]), where they assume the absence of stretch. Based on Eq. (1), simple, mutually exclusive assumptions for the nature of the deformation of the tube segment can be considered:

(1) The tube length changes according to affine deformation and the tube diameter adapts according to Eq. (4). This hypothesis basically suggests affine deformation of the primitive path. We call this assumption "affine stretching".

(2) The tube diameter changes according to affine deformation and the local stretch ratio $\lambda$ adapts through Eq. (4). We name this assumption "affine squeezing".

In this work, two independent full tensorial constitutive models are developed from the above assumptions. Using Eqs. (7) and (20) of Marrucci and Ianniruberto [1], an evolution equation for $\alpha$ based on the affine stretching assumption can be written as follows:

$\dot{\alpha}=-\dot{\varepsilon} \alpha+\frac{1}{\tau_{\mathrm{p}}}\left(\alpha^{-3}-1\right)$.

The first term on the right hand side of Eq. (5) is the convection term, while the second term describes relaxation toward equilibrium driven by the compression force. Similarly, an evolution equation for $\alpha$ based on the affine squeezing assumption can be written as:

$\dot{\alpha}=-\frac{\dot{\varepsilon}}{2} \alpha+\frac{1}{\tau_{\mathrm{p}}}\left(\alpha^{-3}-1\right)$.

The parameter $\tau_{\mathrm{p}}$ is the characteristic time required for the tube segment to come back to its equilibrium (diameter) state from its deformed state. It should be mentioned here that the exact expression for $\tau_{\mathrm{p}}$ is not known, but it scales like $\tau_{\mathrm{r}}$ [1]. Eq. (6) differs from Eq. (20) in [1] by the factor 0.5 in front of the strain rate. This factor reflects the assumption of affine shrinking of the tube diameter (in the absence of relaxation phenomena). By ignoring it, Marrucci and Ianniruberto implicitly choose the affine stretching assumption (Eq. (5) in the present work). The relevance of the factor of 0.5 will be shown later, when both assumptions are generalized to a full constitutive equation, since its inclusion or exclusion yields different strain measures.

Making use of Eq. (4), the equation for the evolution of $\lambda$ for the affine stretching case is given as:

$\dot{\lambda}=\dot{\varepsilon} \lambda-\frac{1}{\tau_{\mathrm{p}}}\left(\lambda^{5}-\lambda^{2}\right)$.

So far, this simple model, written using $\lambda$ is totally equivalent to Marrucci and Ianniruberto [1]. In order to incorporate finite extensibility into this model, the method proposed by Rolon-Garrido et al. [22], has been followed. Therefore, the modified evolution equation for $\lambda$ is given as follows:

$\dot{\lambda}=\dot{\varepsilon} \lambda-\frac{1}{\tau_{\mathrm{p}}}\left(\left(f\left(\lambda^{2}\right)\right)^{2} \lambda^{5}-\lambda^{2}\right)$.

The finite extensibility modification to the stiffness of the entropic spring is computed as for a FENE spring:

$f\left(\lambda^{2}\right)=\frac{\lambda_{\max }^{2}-1}{\lambda_{\max }^{2}-\lambda^{2}}$,

where $\lambda_{\max }$ is of the order of the square root of the number of Kuhn steps in the tube segment.

Similarly, for the affine squeezing case we get:

$\dot{\lambda}=\frac{\dot{\varepsilon}}{2} \lambda-\frac{1}{\tau_{\mathrm{p}}}\left(\left(f\left(\lambda^{2}\right)\right)^{2} \lambda^{5}-\lambda^{2}\right)$

It has to be kept in mind that, for a given affine deformation of the tube radius characterized by a ratio $\alpha$, the generated stretch level $\lambda=\alpha^{-1}$ is only the square root of the level that would be generated if everything was deforming affinely (i.e., $\lambda=\alpha^{-2}$ ).

\section{Building a single segment model}

This section is dedicated to the building of a full tensorial, single segment constitutive model. As discussed above, the model is developed based on either one of the alternative assumptions i.e., affine squeezing or affine stretching.

The first step is to choose a set of structural variables and assign them a clear meaning. Let us call $\mathbf{R}$ the vector of norm $\lambda$ tangent to a given tube segment. The average orientation and stretch of the microstructure at a given time can therefore be described through the tensor $\mathbf{B}$ defined as:

$\boldsymbol{B}=3\langle\boldsymbol{R R}\rangle$.

By using Kramers formula [26], an expression for the extra stress tensor can be obtained:

$\sigma=G_{\mathrm{N}}^{0}(f(\operatorname{tr} \boldsymbol{B}) \boldsymbol{B}-\delta)$,

with $f(\operatorname{tr} \mathbf{B})$ defined as:

$f=\frac{b-3}{b-\operatorname{tr} \boldsymbol{B}}$

and $b=3 \lambda_{\max }^{2}$.

The value of the maximum square stretch $\lambda_{\max }^{2}$ can be estimated as the number of Kuhn steps between entanglements. In the following sections, we will show how we can use the affine stretching and affine squeezing assumptions to derive an evolution equation for $\mathbf{B}$. 


\subsection{Affine stretching model}

If tube segments orient and stretch affinely under a step strain, the evolution equation for $\mathbf{B}$ in the absence of relaxation mechanisms simply writes:

$\stackrel{\nabla}{\boldsymbol{B}}=0$,

where $\stackrel{\nabla}{B}$ is the upper convected derivative of $\mathbf{B}$. According to the definition of $\mathbf{B}$ (Eq. (11)), the trace of $\mathbf{B}$ can be related to the average of $3 \lambda^{2}$. Multiplying Eq. (8) by $2 \lambda$, the evolution equation for $\lambda^{2}$ is obtained:

$\dot{\lambda}^{2}=2 \dot{\varepsilon} \lambda^{2}-\frac{2}{\tau_{\mathrm{p}}}\left(f^{2} \lambda^{6}-\lambda^{3}\right)$.

Since, the trace of $\mathbf{B}$ is related to the average of $3 \lambda^{2}$, the trace of a full tensorial model should produce Eq. (15). So, we propose the following compatible full tensorial constitutive equation (Eq. (16)).

$\stackrel{\nabla}{\boldsymbol{B}}=-\frac{2}{9 \tau_{\mathrm{p}}}\left(f(\operatorname{tr} \boldsymbol{B})^{2} \operatorname{tr}\left(\boldsymbol{B}^{3}\right)-\operatorname{tr} \boldsymbol{B}\right) \frac{\boldsymbol{B}}{\operatorname{tr} \boldsymbol{B}}$.

As it should, the right hand side of the above equation vanishes at equilibrium. With the assumption of $\lambda^{3} \approx \lambda^{2} / 9$, the trace of Eq. (16) produces Eq. (15). The assumption, $\lambda^{3} \approx \lambda^{2} / 9$ is used in order to reduce the level of non-linearity in the model. The sensitivity of this assumption has been tested, and it has been found that it makes no difference on the predictions. In Eq. (16), we could have used $\lambda^{6} \sim$ $(\operatorname{tr} \mathbf{B})^{3}$ instead of $\operatorname{tr} \mathbf{B}^{3}$. We have tested the two alternatives and found no difference in the predictions for all the systems analyzed in the paper.

We can assume that the relaxation of $\mathbf{B}$ is simply a superposition of different relaxation mechanisms happening at different time scales. This allows us to add "reptation dynamics" and "stretch dynamics" in the following way:

$$
\begin{aligned}
\stackrel{\nabla}{\boldsymbol{B}}= & -\frac{2}{9 \tau_{\mathrm{p}}}\left(f(\operatorname{tr} \boldsymbol{B})^{2} \operatorname{tr}\left(\boldsymbol{B}^{3}\right)-\operatorname{tr} \boldsymbol{B}\right) \frac{\boldsymbol{B}}{\operatorname{tr} \boldsymbol{B}}-\frac{1}{\tau_{\mathrm{d}}}(f(\operatorname{tr} \boldsymbol{B}) \boldsymbol{B}-\delta) \\
& -\frac{1}{\tau_{\mathrm{r}}}(f(\operatorname{tr} \boldsymbol{B}) \operatorname{tr} \boldsymbol{B}-3) \frac{\boldsymbol{B}}{\operatorname{tr} \boldsymbol{B}} .
\end{aligned}
$$

Similarly, convective constraint release can be included by modifying the reptation time $\tau_{\mathrm{d}}$. The modified or effective reptation rate $\left(1 / \tau_{\text {eff }}\right)$ is basically a superposition of the reptation rate $\left(1 / \tau_{d}\right)$, and the rate of convection of the entanglements by stretch dynamics $\left(1 / \tau_{\mathrm{r}}\right)$ and tube diameter dynamics $\left(1 / \tau_{\mathrm{p}}\right)$. Basically, it is added in a similar fashion as proposed by Marrucci and Ianniruberto [21] in their 2D model for interchain tube pressure effects:

$$
\begin{aligned}
\stackrel{\nabla}{\boldsymbol{B}}= & -\frac{2}{9 \tau_{\mathrm{p}}}\left(f(\operatorname{tr} \boldsymbol{B})^{2} \operatorname{tr}\left(\boldsymbol{B}^{3}\right)-\operatorname{tr} \boldsymbol{B}\right) \frac{\boldsymbol{B}}{\operatorname{tr} \boldsymbol{B}} \\
& -\frac{1}{\tau_{\mathrm{eff}}}(f(\operatorname{tr} \boldsymbol{B}) \boldsymbol{B}-\delta)-\frac{1}{\tau_{\mathrm{r}}}(f(\operatorname{tr} \boldsymbol{B}) \operatorname{tr} \boldsymbol{B}-3) \frac{\boldsymbol{B}}{\operatorname{tr} \boldsymbol{B}},
\end{aligned}
$$

where

$$
\frac{1}{\tau_{\text {eff }}}=\frac{1}{\tau_{\mathrm{d}}}+\beta\left[\frac{2}{9 \tau_{\mathrm{p}}}\left(f(\operatorname{tr} \boldsymbol{B})^{2} \operatorname{tr}\left(\boldsymbol{B}^{3}\right)-\operatorname{tr} \boldsymbol{B}\right)+\frac{1}{\tau_{\mathrm{r}}}(f(\operatorname{tr} \boldsymbol{B}) \operatorname{tr} \boldsymbol{B}-3)\right] .
$$

The adjustable numerical coefficient $\beta$ weighs the importance of convective constraint release.

\subsection{Affine squeezing model}

If we assume affine squeezing and instantaneous equilibration of the primitive path length according to the tube diameter, the stretching of the microstructure is non-affine. More precisely, after an instantaneous deformation characterized by a deformation tensor $\mathbf{E}$, the new tube vector $\mathbf{R}^{\prime}$ is given by the following expression:

$\boldsymbol{R}^{\prime}=\frac{\boldsymbol{E} \cdot \boldsymbol{R}}{\sqrt{|\boldsymbol{E} \cdot \boldsymbol{R}|}}$.

The expression for $\mathbf{B}$ as a function of $\mathbf{E}$ now becomes:

$\boldsymbol{B}=3\left\langle\frac{\boldsymbol{E} \cdot \boldsymbol{u} \boldsymbol{E} \cdot \boldsymbol{u}}{|\boldsymbol{E} \cdot \boldsymbol{u}|}\right\rangle$,

where $\mathbf{u}$ is a orientation vector distributed on the unit sphere. This expression for $\mathbf{B}$ is very closely related to the $\mathbf{Q}$ tensor of Doi and Edwards [2] (without independent alignment). Following the previous work of Marrucci and Greco [24] on alternative strain measures based on force balance arguments, we choose to approximate $\mathbf{B}$ after a step strain with the (matrix) square root of the Finger tensor corresponding to the deformation characterized by $\mathbf{E}$ :

$\boldsymbol{B}=(3\langle\boldsymbol{E} \cdot \boldsymbol{u} \boldsymbol{E} \cdot \boldsymbol{u}\rangle)^{1 / 2} \approx 3\left\langle\frac{\boldsymbol{E} \cdot \boldsymbol{u} \boldsymbol{E} \cdot \boldsymbol{u}}{|\boldsymbol{E} \cdot \boldsymbol{u}|}\right\rangle$.

This simple approximation for $\mathbf{B}$ has the following properties:

- The principal orientations, i.e., the eigenvectors of the approximations correspond to those of an affine deformation.

- The average stretch level is only the square root of the stretch level of an affine deformation.

- Following a step strain in shear, this tensor will predict a second normal stress difference.

- This tensor yields a strain measure which can be implemented in a differential models [21,25].

Let us define $\mathbf{C}$ as the (matrix) square of $\mathbf{B}$ :

$\boldsymbol{C}=\boldsymbol{B}^{2}$.

When relaxation phenomena can be considered as frozen, the tensor $\mathbf{C}$ evolves according to the upper convected derivative and is, therefore, an appealing choice as a variable.

For the affine squeezing assumption (Eq. (10), the trace of $\mathbf{C}$ is related to the average of $9 \lambda^{4}$. Therefore, multiplying Eq. (10) by $4 \lambda^{3}$, the evolution equation for $\lambda^{4}$ is given as follows:

$\dot{\lambda}^{4}=2 \dot{\varepsilon} \lambda^{4}-\frac{4}{\tau_{\mathrm{p}}}\left(f^{2} \lambda^{8}-\lambda^{5}\right)$.

So, by assuming $\lambda^{5} \sim \lambda^{4} / 9$, and following the same steps as that for the affine stretching model, the full constitutive equation for affine squeezing assumption can be obtained:

$$
\begin{aligned}
\stackrel{\nabla}{\boldsymbol{C}}= & -\frac{4}{9 \tau_{\mathrm{p}}}\left(f\left(\operatorname{tr} \boldsymbol{C}^{1 / 2}\right)^{2} \operatorname{tr}\left(\boldsymbol{C}^{2}\right)-\operatorname{tr} \boldsymbol{C}\right) \frac{\boldsymbol{C}}{\operatorname{tr} \boldsymbol{C}}-\frac{2}{\tau_{\mathrm{eff}}}\left(f\left(\operatorname{tr} \boldsymbol{C}^{1 / 2}\right) \boldsymbol{C}-\boldsymbol{C}^{1 / 2}\right) \\
& -\frac{1}{\tau_{\mathrm{r}}}\left(f\left(\operatorname{tr} \boldsymbol{C}^{1 / 2}\right) \operatorname{tr} \boldsymbol{C}-3\right) \frac{\boldsymbol{C}}{\operatorname{tr} \boldsymbol{C}}
\end{aligned}
$$

where,

$$
\begin{aligned}
\frac{1}{\tau_{\mathrm{eff}}}= & \frac{1}{\tau_{\mathrm{d}}} \\
& +\beta\left[\frac{4}{9 \tau_{\mathrm{p}}}\left(f\left(\operatorname{tr} \boldsymbol{C}^{1 / 2}\right)^{2} \operatorname{tr}\left(\boldsymbol{C}^{2}\right)-\operatorname{tr} \boldsymbol{C}\right)+\frac{1}{\tau_{\mathrm{r}}}\left(f\left(\operatorname{tr} \boldsymbol{C}^{1 / 2}\right) \operatorname{tr} \boldsymbol{C}-3\right)\right]
\end{aligned}
$$

In the above equation $\stackrel{\nabla}{\boldsymbol{C}}$ is an upper convected derivative on $\mathbf{C}$ and $f\left(\mathbf{C}^{1 / 2}\right)$ is defined as follows:

$\left.f(\operatorname{trC})^{1 / 2}\right)=\frac{b-3}{b-\operatorname{tr} C^{1 / 2}}$,

with $b=3 \lambda_{\max }^{2}$. 


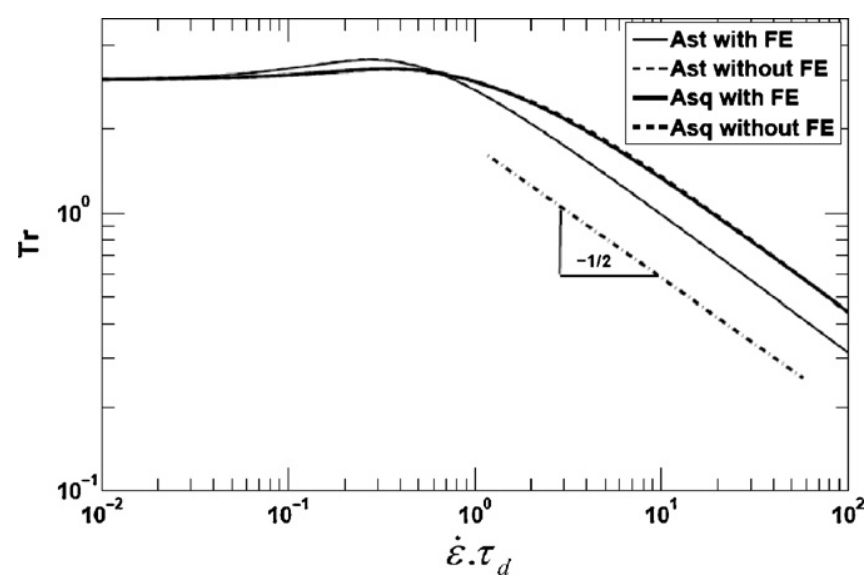

Fig. 2. Trouton ratio ( $\mathrm{Tr}$ ) vs. non-dimensional rate of elongation in uniaxial elongational flow: Effect of finite extensibility in Zone I ( $\tau_{\mathrm{d}} / \tau_{\mathrm{p}}=0.1, \tau_{\mathrm{r}} \rightarrow \infty, b \rightarrow \infty$ (no FE), $b=66$ (with FE), $\beta=0$ ).

In terms of the variable $\mathbf{C}$, the extra stress tensor writes:

$\sigma=2 G_{\mathrm{N}}^{0}\left(f\left(t r \boldsymbol{C}^{1 / 2}\right) \boldsymbol{C}^{1 / 2}-\delta\right)$.

\section{Model parameters}

The material parameters required for the above proposed models are, a reptation time, $\tau_{\mathrm{d}}$, a Rouse relaxation time, $\tau_{\mathrm{r}}$, a tube relaxation time, $\tau_{p}$, a plateau modulus, $G_{\mathrm{N}}^{0}$, and a finite extensibility factor, $b$. All parameters, except $\tau_{\mathrm{p}}$, can be determined based on LVE data or structural information on the polymer. For qualitative predictions, $\tau_{\mathrm{p}} / \tau_{\mathrm{d}}=10$ is used to represent moderately entangled systems (Zone I in Fig. 1 ) and $\tau_{\mathrm{p}} / \tau_{\mathrm{d}}=0.1$ for highly entangled systems (Zone II in Fig. 1 ). We take $\tau_{\mathrm{r}}=\tau_{\mathrm{p}} / 100$ in both cases. This scaling is consistent with Marrucci and Ianniruberto [1]. For quantitative predictions, the reptation time $\tau_{\mathrm{d}}$ is determined as $\tau_{\mathrm{d}}=\eta_{0} / G_{\mathrm{N}}^{0}$. Rouse time values for two polystyrene samples (PS200 and PS390) are taken from Marrucci and Ianniruberto [1]. The tube pressure relaxation time $\tau_{\mathrm{p}}$ is adjusted manually in order to fit the non-LVE data. Though the exact expression for $\tau_{\mathrm{p}}$ is not known, it is known to scale as $\tau_{\mathrm{r}}\left(\sim M^{2}\right)$ [1]. This manually adjusted value of $\tau_{p}$ is then justified by comparing the scaling of $\tau_{p}$ with molar mass. The value of $b$ is determined based on molecular structure of the polymer, e.g., for polystyrene $b=66\left(=3 N_{\mathrm{k}}\right.$, where $N_{\mathrm{k}}$ is number of Kuhn steps) [27]. For the Gaussian limit, we have $b \rightarrow \infty$.

\section{Results and discussion}

This section presents qualitative and quantitative predictions by both models for moderately entangled polymer systems (Zone I, Fig. 1). Because of the lack of reliable non-linear data for highly entangled monodisperse systems, predictions are restricted to a qualitative analysis in Zone II. Different observations and comparisons with other relevant differential tube models are discussed in each subsection.

\subsection{Qualitative predictions}

\subsubsection{Elongational flow}

5.1.1.1. Effect of finite extensibility. Fig. 2 shows the normalized steady state elongational viscosity $\left(\operatorname{Tr}=\eta_{\mathrm{el}} / \eta_{0}\right)$ as a function of elongational rate for both models, with $(b=66)$ and without $(b \rightarrow \infty)$ finite extensibility, in Zone I. In order to see the effect of finite extensibility on ITPE, we use the values $\tau_{\mathrm{p}} / \tau_{\mathrm{d}}=10, \tau_{\mathrm{r}} \rightarrow \infty$, and $\beta=0$ in order to make CCR inactive. In Fig. 2, and also in the following figures, the abbreviations 'Ast' and 'Asq' stand for affine stretching

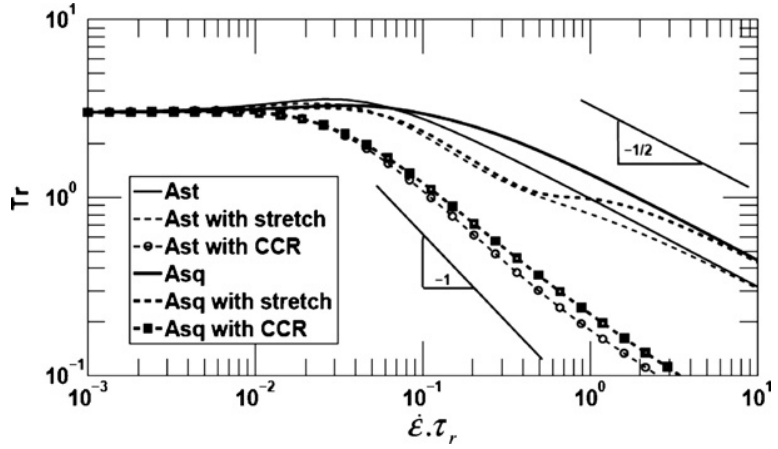

Fig. 3. Trouton ratio ( $\mathrm{Tr}$ ) vs. non-dimensional rate of elongation in uniaxial elongational flow: Effect of stretch dynamics and CCR in Zone I $\left(\tau_{\mathrm{d}} / \tau_{\mathrm{p}}=0.1, \tau_{\mathrm{r}}=\tau_{\mathrm{p}} / 100\right.$, $b \rightarrow \infty, \beta=1)$.

model and affine squeezing model, respectively. Fig. 2 clearly shows that finite extensibility is not playing any role in elongational flows in steady state conditions. In transient conditions, finite extensibility plays some role at high elongational rates, though the difference becomes less significant while moving from affine squeezing to affine stretching and also with the inclusion of stretch dynamics.

The same observations about the role of finite extensibility apply to Zone II. Therefore, the "infinite extensibility" limit $(b \rightarrow \infty)$ has been used for the remainder of this work.

5.1.1.2. Effect of Stretch and CCR. Fig. 3 shows the effect of stretch dynamics $\left(\tau_{\mathrm{r}}=\tau_{\mathrm{p}} / 100, \beta=0\right)$ and CCR $\left(\tau_{\mathrm{r}}=\tau_{\mathrm{p}} / 100, \beta=1\right)$ on the steady state elongational viscosity for both models in Zone I. With the inclusion of stretch dynamics, there is no upturn of the steadystate elongational viscosity at $\dot{\varepsilon} \sim 1 / \tau_{\mathrm{r}}$ (as in classical tube theories, see Fig. 4), but there is an upward kink, after which $\eta_{\mathrm{el}}$ vs. $\dot{\varepsilon}$ follows a -0.5 power law. The power law observed for $\eta_{\mathrm{el}}$ between $1 / \tau_{\mathrm{d}}$ and $1 / \tau_{\mathrm{r}}$ is (approximately) -1 , which is in line with classical tube theories (see Fig. 4). It will be shown in the section on quantitative predictions that these features are all consistent with experimental observations [17].

Inclusion of CCR causes more elongational thinning. Although the effect of CCR should also be tested in shear, it is obvious that CCR does not improve predictions in elongational flows, as will be confirmed by the quantitative comparisons (Section 5.2). The predictions of the models in Zone II are qualitatively similar to those in Zone I.

5.1.1.3. Comparisons with other Models. Fig. 4 compares the predictions of the new models with the predictions of coupled DCR [9], Rolie-Poly [14], and CRAFT [15] for steady state elongational viscos-

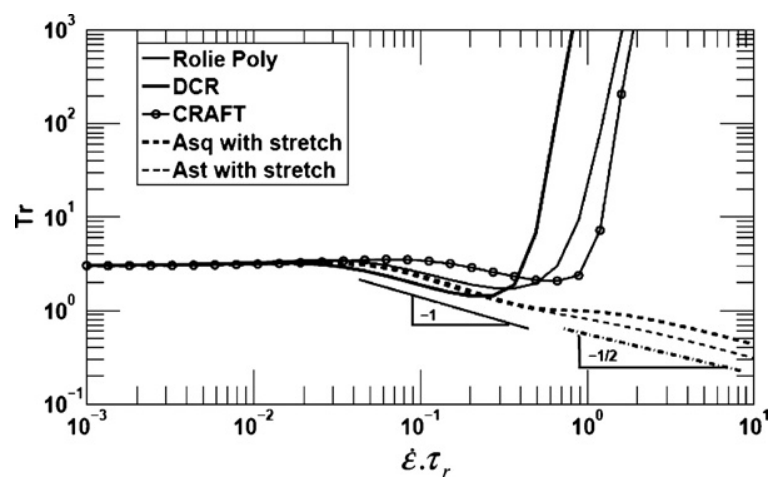

Fig. 4. Trouton ratio ( $\mathrm{Tr})$ vs. non-dimensional rate of elongation in uniaxial elongational flow: Comparison between models in Zone $\mathrm{I}\left(\tau_{\mathrm{d}} / \tau_{\mathrm{p}}=0.1, \tau_{\mathrm{r}}=\tau_{\mathrm{p}} / 100, b \rightarrow \infty\right.$, $\beta=0)$. 

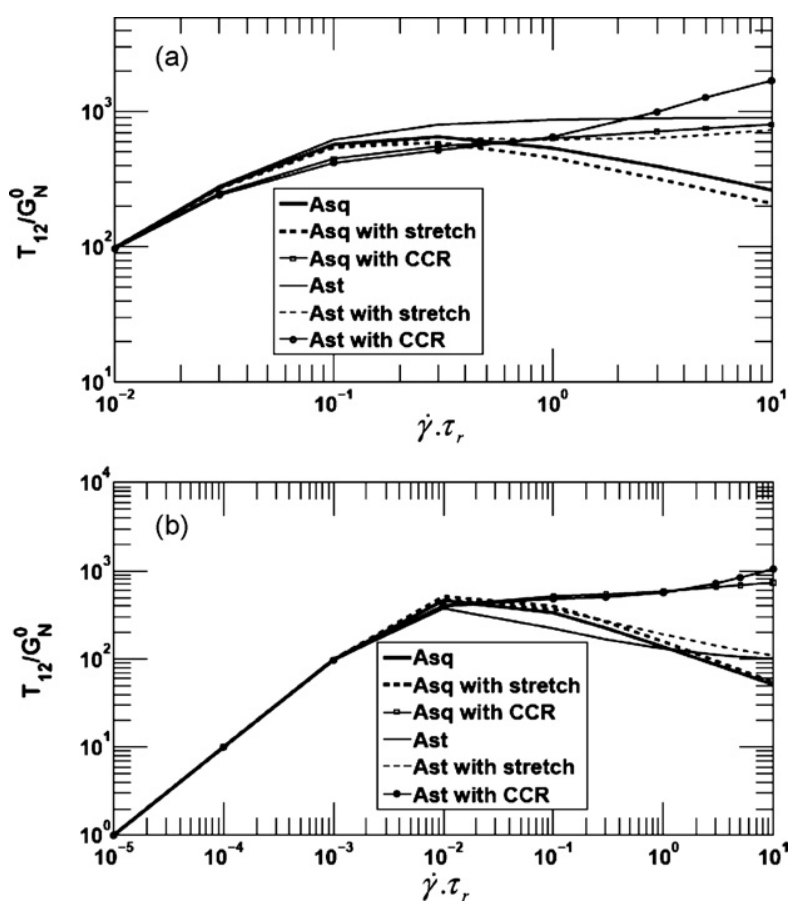

Fig. 5. Non-dimensional steady state shear stress vs. non-dimensional shear rate: (a) Zone I $\left(\tau_{\mathrm{d}} / \tau_{\mathrm{p}}=0.1, \tau_{\mathrm{r}}=\tau_{\mathrm{p}} / 100, b \rightarrow \infty, \beta=1\right)$, and (b) Zone II $\left(\tau_{\mathrm{d}} / \tau_{\mathrm{p}}=10, \tau_{\mathrm{r}}=\tau_{\mathrm{p}} / 100\right.$, $b \rightarrow \infty, \beta=1)$.

ity. The model parameters used are, Gaussian chain limit $(b \rightarrow \infty)$, $\tau_{\mathrm{r}}=\tau_{\mathrm{p}} / 100, \tau_{\mathrm{d}} / \tau_{\mathrm{p}}=0.1$, and $\beta=0$ (no CCR). Predictions by other models show strong elongational thickening at $\dot{\varepsilon}=1 / \tau_{\mathrm{r}}$, while both affine squeezing and affine stretching models show elongational thinning even above $\dot{\varepsilon}>1 / \tau_{\mathrm{r}}$, except a small upward kink around $\dot{\varepsilon}=1 / \tau_{\mathrm{r}}$. The qualitative behaviour of the proposed models is clearly superior.

\subsubsection{Shear flow}

5.1.2.1. Effect of Stretch and CCR. As indicated in the above section, the Ast and Asq models show qualitatively similar predictions for elongational flow in Zones I and II. The effect of stretch dynamics and CCR on steady state shear predictions is summarized in Fig. 5. Fig. 5(a) shows the non-dimensional steady state shear stress $\left(T_{12} / G_{\mathrm{N}}^{0}\right)$ vs. Deborah number $\left(\tau_{\mathrm{r}}\right.$-based $)$ for Zone I $\left(\tau_{\mathrm{d}} / \tau_{\mathrm{p}}=0.1\right)$. It can be observed that at high shear-rates, the affine squeezing model predicts unstable shear-thinning behaviour in the absence of CCR $(\beta=0)$, but the affine stretch model is always stable, even without CCR. By reference to the elongational flow predictions presented above, this observation leads to the conclusion that the affine stretching model may be physically more meaningful than the affine squeezing model and that CCR is not needed for stability in the case of moderately entangled systems. On the other hand, as seen from Fig. 5 (b) for Zone II $\left(\tau_{\mathrm{d}} / \tau_{\mathrm{p}}=10\right)$ i.e., for highly entangled systems, CCR is needed for stability in both models. The inclusion of stretch dynamics qualitatively does not make a difference in both zones and for both models, but it improves the quantitative predictions, as will be seen in the next section.

5.1.2.2. Comparisons with other models. Comparisons between the predictions of the Ast, Asq, and other models [9,14,15] for steady state shear stress are shown in Fig. 6. All predictions are made for the no CCR condition $(\beta=0)$. It is seen that all models except the affine stretching model show a maximum shear stress. The maximum steady state shear stress shown by Rolie-Poly is not very pronounced despite the absence of CCR, supporting the idea that CCR may not be important in moderately entangled systems. The

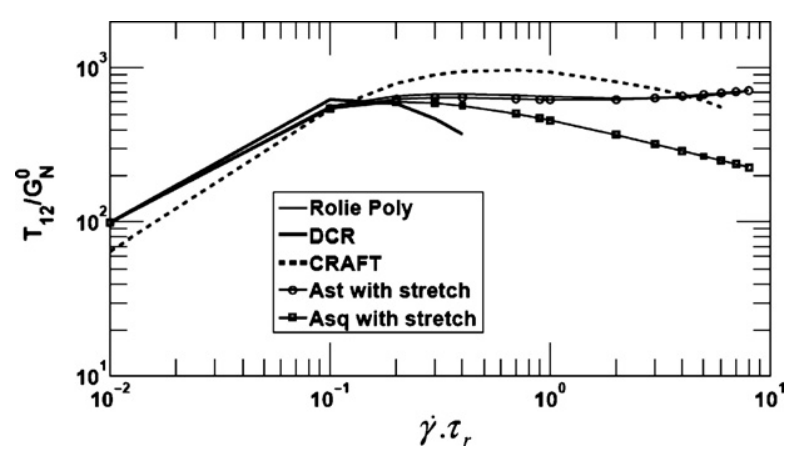

Fig. 6. Non-dimensional steady state shear stress vs. non-dimensional shear rate: comparison between models in Zone $\mathrm{I}\left(\tau_{\mathrm{d}} / \tau_{\mathrm{p}}=0.1, \tau_{\mathrm{r}}=\tau_{\mathrm{p}} / 100, b \rightarrow \infty, \beta=0\right)$.

non relevance of CCR at high shear rates has also been supported by recent slip-link simulations of Schieber et al. [28] for moderately entangled PS systems. This conclusion may not be valid for highly entangled systems as the importance of CCR may depend on the level of entanglements (see Fig. 5(b)).

\subsection{Quantitative predictions}

Data of Bach et al. [17] and Schweizer et al. [29] in elongational and shear flow, respectively, have been used for quantitative predictions. The samples (polystyrene, $Z \sim 20, \tau_{\mathrm{d}} \approx \tau_{\mathrm{p}}$ ) fall in the moderately entangled category.

\subsubsection{Elongational flow}

Figs. 7 and 8 show the comparisons of the affine squeezing and affine stretching models predictions with the elongational data of Bach et al. [17] for two samples (PS200 and PS390). The model parameters used are given in Table 1.

The ratio $\tau_{\mathrm{p}}$ for PS390 to $\tau_{\mathrm{p}}$ for PS200 is 3.76, and the ratio $M^{2}$ for PS390 to $M^{2}$ for PS200 is 3.8. Thus, the molar mass scaling of $\tau_{\mathrm{p}}$ as given by Marrucci and Ianniruberto [1] is about right. It should also be noted that $\tau_{\mathrm{p}} \sim 10 \tau_{\mathrm{r}}$, which is also consistent with Marrucci and Ianniruberto [1].

5.2.1.1. Transient flow. Figs. 7(a, c) and 8(a, c) show the effect of stretch dynamics and CCR on the transient tensile stress as a function of Hencky strain $\varepsilon$, for PS200 and PS390. It can be seen from these Figs. (7(a, c), 8(a, c)) that the model predictions are good, especially for affine stretching. Inclusion of stretch dynamics improves the predictions by both models, but inclusion of CCR works detrimentally.

5.2.1.2. Steady flow. Figs. 7 (b, d) and 8 (b, d) show the variation of the Trouton ratio $\left(\eta_{\mathrm{el}} / \eta_{0}\right)$ vs. elongational rate $\dot{\varepsilon}$ for PS200 and PS390. Predictions by both models can be compared with the experimental data. In the relaxation zone, both models, when only including reptation and tube pressure terms (i.e., $\tau_{\mathrm{r}} \approx \infty$ and $\beta=0$ ), follow the -0.5 power law for $\eta_{\mathrm{el}} \mathrm{vs} . \dot{\varepsilon}$. Inclusion of stretch dynamics gives an additional small upward kink at the transition (around $\dot{\varepsilon}=1 / \tau_{\mathrm{r}}$ ) from -1 power law to -0.5 power law. The entire feature is consistent with the experimental observations (see Figs. $7(\mathrm{~b}, \mathrm{~d})$ and $8(\mathrm{~b}, \mathrm{~d})$ ). Inclusion of $\mathrm{CCR}(\beta=1)$ works detrimentally.

Table 1

Model parameters for PS200 and PS390.

\begin{tabular}{llccr}
\hline Sample & $G_{\mathrm{N}}^{0}(\mathrm{kPa})$ & $\tau_{\mathrm{d}}(\mathrm{s})$ & $\tau_{\mathrm{r}}(\mathrm{s})$ & $\tau_{\mathrm{p}}(\mathrm{s})$ \\
\hline PS200 at $130^{\circ} \mathrm{C}$ & 200 & 428 & 50 & 476 \\
PS390 at $130^{\circ} \mathrm{C}$ & 200 & 3620 & 200 & 1787 \\
PS200 at $175^{\circ} \mathrm{C}$ & 200 & 0.39 & 0.046 & 0.43 \\
\hline
\end{tabular}



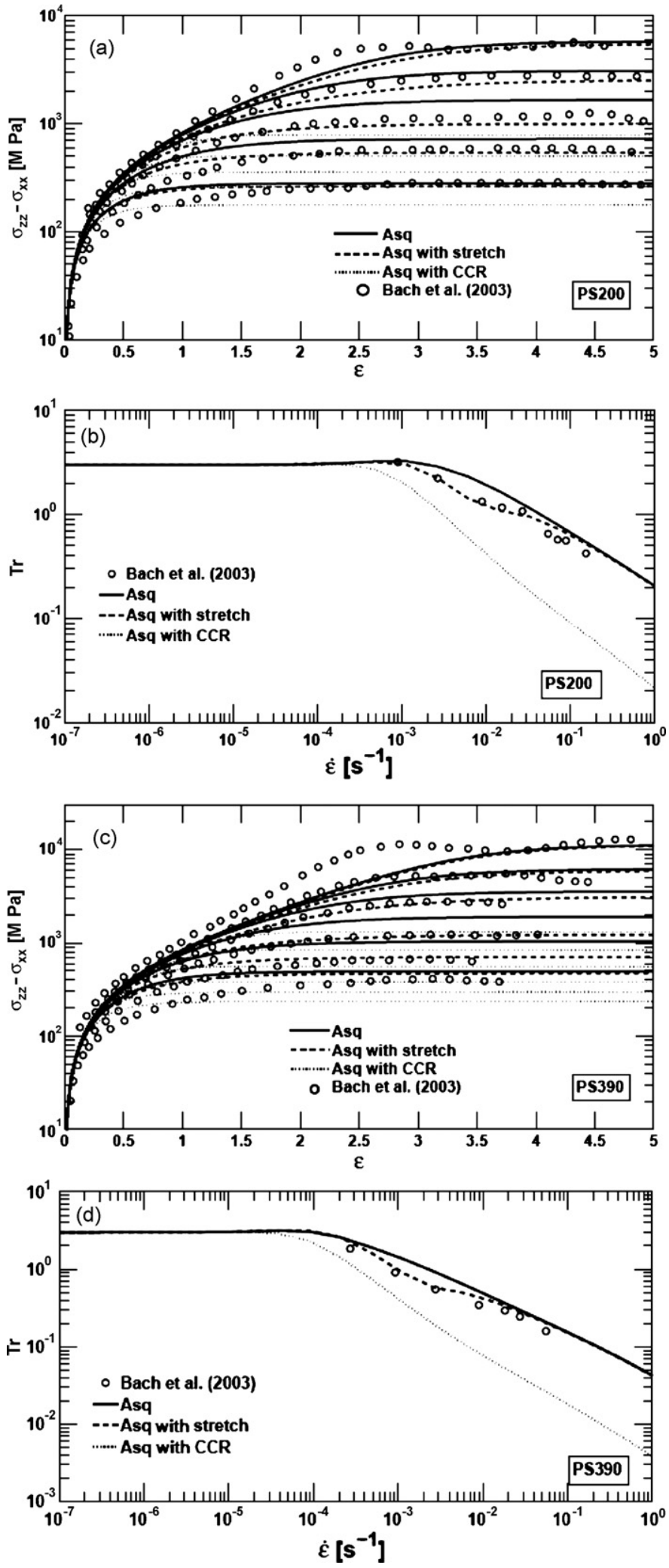

Fig. 7. Comparisons between experimental data [17] on transient tensile stress (a and c) and steady state elongational viscosity (b and d) and predictions by the affine squeezing model. (The symbols from top to bottom are experimental data for elongation rate $(\dot{\varepsilon})$ of $0.1,0.03,0.01,0.003$ and $0.001 \mathrm{~s}^{-1}$ for PS200 and for elongation rate $(\dot{\varepsilon})$ of $0.1,0.03,0.01,0.003,0.001$ and $0.0003 \mathrm{~s}^{-1}$ for PS390 in a and c, respectively.)
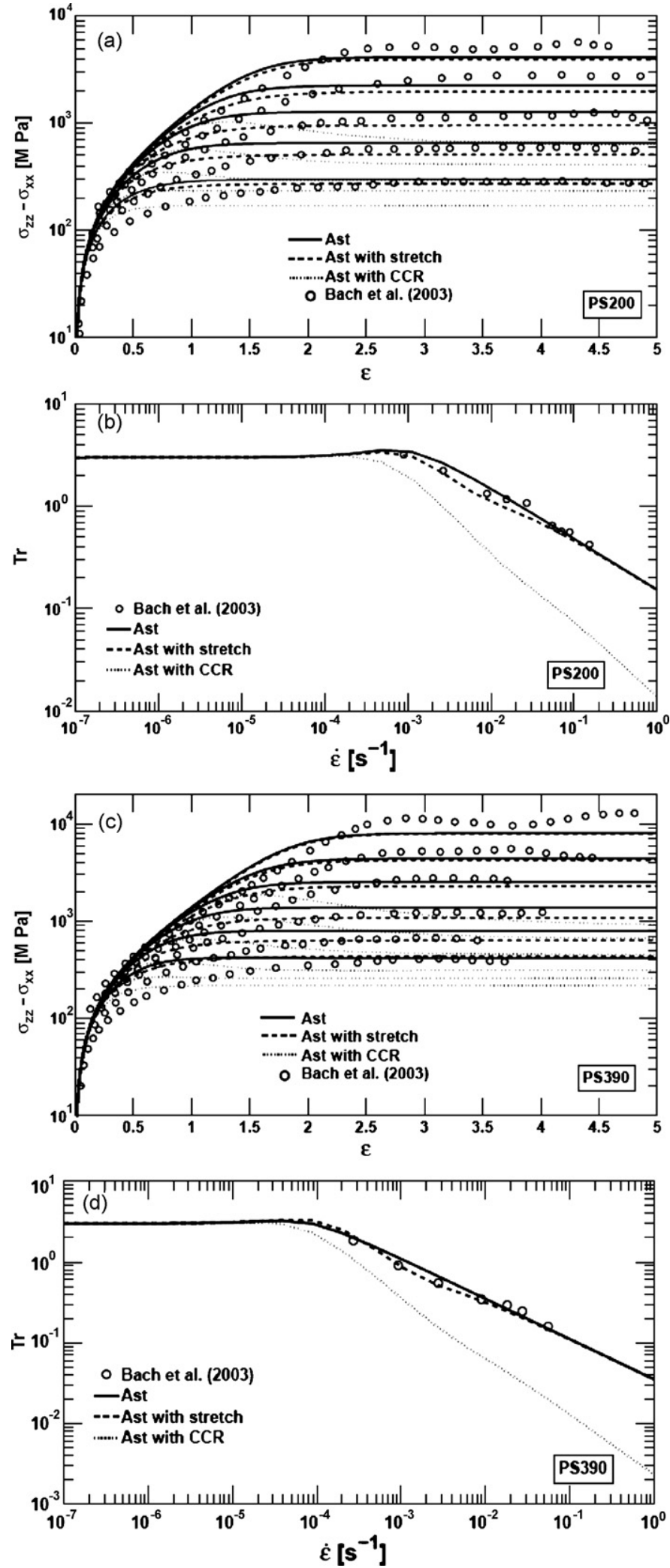

Fig. 8. Comparisons between experimental data [17] on transient tensile stress (a and c) and steady state elongational viscosity (b and d) and predictions by the affine stretching model. (The symbols from top to bottom are experimental data for elongation rate $(\dot{\varepsilon})$ of $0.1,0.03,0.01,0.003$ and $0.001 \mathrm{~s}^{-1}$ for PS200 and for elongation rate $(\dot{\varepsilon})$ of $0.1,0.03,0.01,0.003,0.001$ and $0.0003 \mathrm{~s}^{-1}$ for PS390 in a and c, respectively.) 


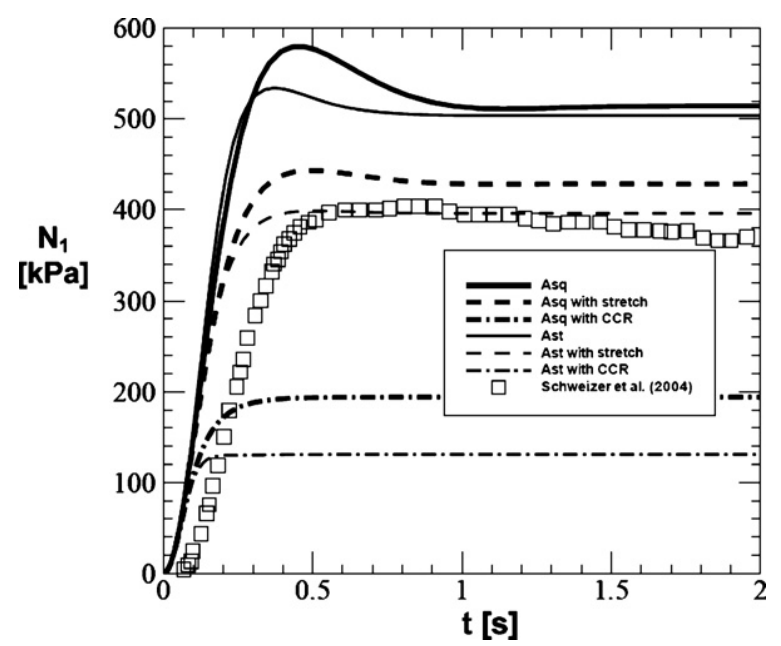

Fig. 9. Comparisons between experimental data [29] on transient first normal stress difference $\left(N_{1}\right)$ and predictions by both the affine squeezing and affine stretching model.

\subsubsection{Shear flow}

Figs. 9-12 show the quantitative predictions of the models for transient and steady shear flow for PS200. Experimental data by Schweizer et al. [29] are used for this purpose. The model parameters used for PS200 (Table 1) are shifted to $175^{\circ} \mathrm{C}$ [17]. The corresponding values are shown in Table 1.

5.2.2.1. Transient flow. Fig. 9 shows the quantitative predictions for the transient first normal stress difference $N_{1}$, at $\dot{\gamma}=10 \mathrm{~s}^{-1}$ for PS200. Both models, when only reptation and tube pressure terms are included (solid lines) show a maximum before reaching steady state. The inclusion of stretch dynamics (dashed lines) almost erases the peaks and also improves the predictions significantly, especially for the affine stretching model. On the other hand, $\operatorname{CCR}(\beta=1$, dotted lines) reduces the steady state values unrealistically. Similar model predictions are obtained for the transient shear stress at $\dot{\gamma}=10 \mathrm{~s}^{-1}$ for PS200 as shown in Fig. 10. As opposed to the dampening of the peaks in the transient $N_{1}$ (Fig. 9), inclusion of the stretch dynamics predicts these peaks nicely in the transient shear stress (Fig. 10). The transient offset between the predictions and the experimental data up to about $0.2 \mathrm{~s}$ is attributed to the axial compliance of the rheometer and the normal force capacity of the transducer [29].

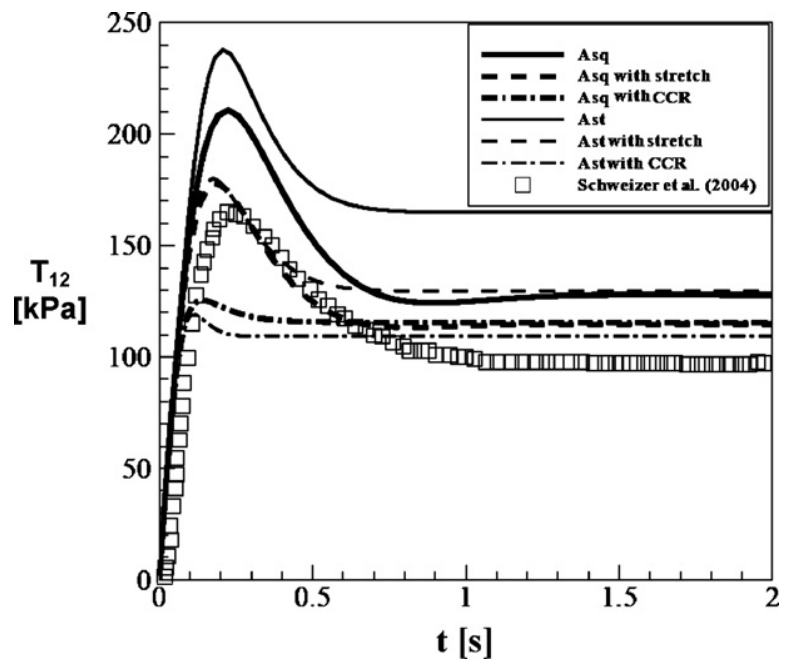

Fig. 10. Comparisons between experimental data [29] on transient shear stress $\left(T_{12}\right)$ and predictions by both the affine squeezing and affine stretching model.

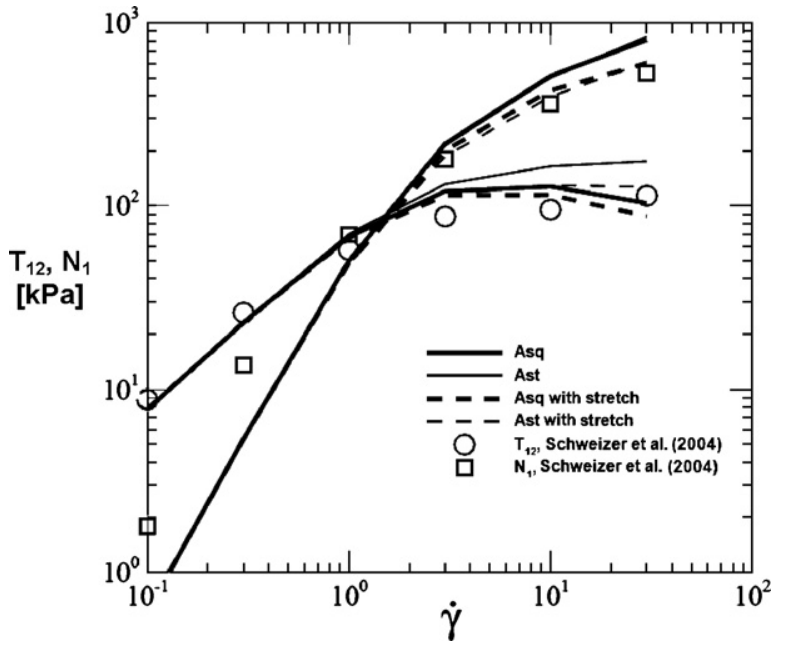

Fig. 11. Comparisons between experimental data [29] on steady state shear stress and first normal stress difference and predictions by both the affine squeezing and affine stretching model.

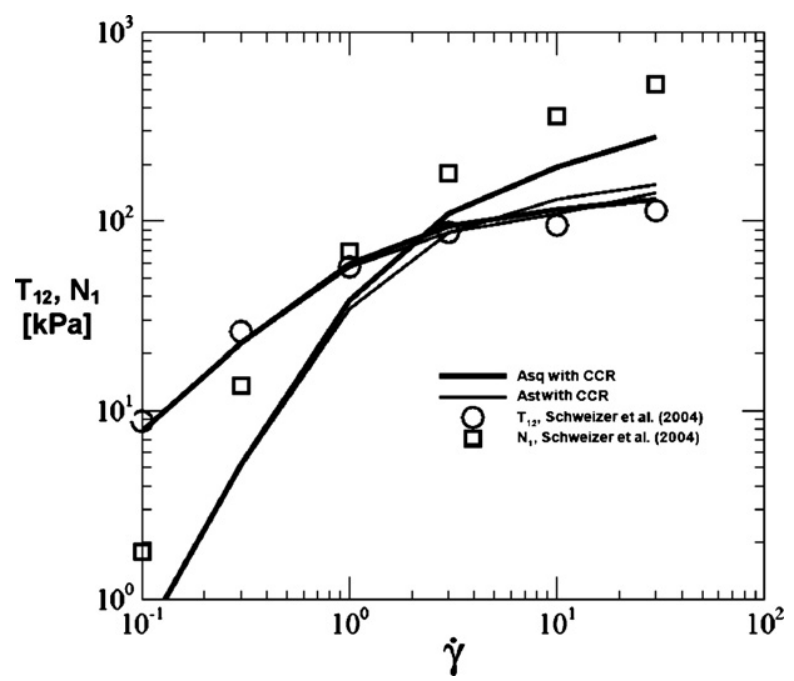

Fig. 12. Effect of CCR on quantitative predictions by both models for steady state shear stress and $N_{1}$.

5.2.2.2. Steady flow. Figs. 11 and 12 show the quantitative steady state predictions for the shear stress T12, and the first normal stress difference $N_{1}$, by both models. It can be seen from Fig. 11 that the predictions by the affine stretching model in the absence of CCR, without (thin solid lines) and with chain stretch (dashed thin lines) are consistent with the experimental data [29]. On the other hand, affine squeezing predicts unstable shear-thinning in both cases at high shear rates. Although inclusion of CCR $(\beta=1)$ stabilizes the affine squeezing model (Fig. 12), the predictions are too much deviating from the experimental data. Thus, CCR seems to be always working detrimentally for moderately entangled systems.

\section{Conclusions}

The simple, single segment models presented in this paper appear promising in describing both qualitatively and quantitatively the non-linear behaviour of entangled linear polymer melts. Principal conclusions of this work are as follow:

- Two simple, single segment tube models, based on either an affine squeezing or an affine stretching assumption have been 
presented. The qualitative and quantitative predictions of both models in shear flow indicate that the affine stretching model is physically more meaningful (stable response in shear flow) than the affine squeezing model for moderately entangled systems (Zone I).

- Predictions in elongational flow by both models (Fig. 2) show that ITPE relaxes the need for unrealistically low finite extensibility.

- Inclusion of the stretch dynamics improves quantitative predictions by both models in elongational and shear flows. Especially, inclusion of stretch dynamics can precisely predict the kink (Figs. 7(b, d) and 8(b, d)) at the transition $\left(\dot{\varepsilon} \sim 1 / \tau_{\mathrm{r}}\right)$ from -1 power law to -0.5 power law, observed by Bach et al. for polystyrene. [17]. This prediction also leads us to consider this experimentally observed kink to be physical.

- Based on the predictions in shear flow (Figs. 9-12), it is concluded that, inclusion of CCR works detrimentally for moderately entangled systems (Zone I) when ITPE is taken into account. This conclusion is consistent with the non importance of CCR observed by Schieber et al. [28] for PS systems in their slip-link simulations. On the other hand, the requirement of CCR for stability (see Fig. 5b) in highly entangled systems (Zone II) leads to the conclusion that CCR may be important in that case.

\section{Acknowledgement}

The authors would like to acknowledge Communauté Française de Belgique for funding this work under the ARC project "Dynema".

\section{References}

[1] G. Marrucci, G. Ianniruberto, Interchain pressure effect in extensional flows of entangled polymers, Macromolecules 36 (2004) 3934-3942.

[2] M. Doi, S.F. Edwards, The Theory of Polymer Dynamics, Clarendon Press, Oxford, 1986.

[3] P.G. De Gennes, Reptation of a polymer chain in the presence of fixed obstacles, J. Chem. Phys. 55 (1971) 572-579.

[4] S.T. Milner, T.C.B. McLeish, Reptation and contour-length fluctuations in melts of linear polymers, Phys. Rev. Lett. 81 (1998) 725-728.

[5] A.E. Likhtman, T.C.B. McLeish, Quantative theory for linear dynamics of linear entangled polymers, Macromolecules 35 (2002) 6332-6343.

[6] E. van Ruymbeke, R. Keunings, V. Stephenne, A. Hagenaars, C. Bailly, Evaluation of reptation models for predicting the linear viscoelastic properties of entangled linear polymers, Macromolecules 35 (2002) 2689-2699.

[7] G. Marrucci, N. Grizzuti, Fast flows of concentrated polymers-predictions of the tube model on chain stretching, Gazz. Chim. Ital. 118 (1988) 179-185.

[8] G. Marrucci, Dynamics of entanglements: a non-linear model consistent with the Cox-Merz rule, J. Non-Newtonian Fluid Mech. 62 (1996) 279-289.

[9] G. Marrucci, G. Ianniruberto, Flow-induced orientation and stretching of entangled polymers, Philos. Trans. R. Soc. London A 361 (2003) 677-688
[10] P. Wapperom, R. Keunings, Impact of decoupling approximation between stretch and orientation in rheometrical and complex flow of entangled polymers, J. Non-Newtonian Fluid Mech. 122 (2004) 33-43.

[11] D.M. Mead, R.G. Larson, M. Doi, A molecular theory for fast flows of entangled polymers, Macromolecules 31 (1998) 7895-7914.

[12] M.H. Wagner, P. Rubio, H. Bastian, The molecular stress function model for polydisperse polymer melts with dissipative convective constraint release, J. Rheol. 45 (2001) 1387-1412.

[13] R.S. Graham, A.E. Likhtman, S.T. Milner, T.C.B. McLeish, Microscopic theory of linear, entangled polymer chains under rapid deformation including chain stretch and convective constraint release, J. Rheol. 47 (2003) 11711200 .

[14] A.E. Likhtman, R.S. Graham, Simple constitutive equation for linear polymer melts derived from molecular theory: Rolie-Poly equation, J. Non-Newtonian Fluid Mech. 114 (2003) 1-12.

[15] A. Leygue, C. Bailly, R. Keunings, A tube based constitutive equation for polydisperse entangled linear polymers, J. Non-Newtonian Fluid Mech. 136 (2006) $1-16$.

[16] J.M. Dealy, R.G. Larson, Structure and Rheology of Molten Polymers: From Structure to Flow behaviour and Back Again, Hanser Gardner, 2006.

[17] A. Bach, K. Almdal, H.K. Rasmussen, O. Hassager, Elongational viscosity of narrow molar mass distribution polystyrene, Macromolecules 36 (2003) 5174-5179.

[18] C. Luap, Ch. Muller, T. Schweizer, D.C. Venerus, Simultaneous stress and birefringence measurements during uniaxial elongation of polystyrene melts with narrow molecular weight distribution, Rheol. Acta 45 (2005) 83-91.

[19] J.K. Nielsen, H.K. Rasmussen, O. Hassager, G.H. McKinley, Elongational viscosity of monodisperse and bidisperse polystyrene melts, J. Rheol. 50 (2006) 453476.

[20] A. Leygue, C. Bailly, R. Keunings, Non-linear flows of well characterized polystyrene melts; simulation and comparison with experiments, J. Rheol. submitted for publication.

[21] G. Marrucci, G. Ianniruberto, A 2D model for tube orientation and tube squeezing in fast flows of polymer melts, J. Non-Newtonian Fluid Mech. 128 (2005) 42-49.

[22] V.H. Rolon-Garrido, M.H. Wagner, C. Luap, T. Schweizer, Modeling non-Gaussian extensibility effects in elongation of nearly monodisperse polystyrene melts, J. Rheol. 50 (2006) 327-340.

[23] M.H. Wagner, V.H. Rolon-Garrido, J.K. Nielsen, H.K. Rasmussen, O. Hassager, A constitutive analysis of transient and steady-state elongational viscosities of bidisperse polystyrene blends, J. Rheol. 52 (2008) 67-86.

[24] G. Marrucci, F. Greco, Simple strain measure for entangled polymers, J. Rheol 44 (4) (2000) 845-854.

[25] A. Leygue, A.N. Beris, R. Keunings, A constitutive equation for entangled linear polymers inspired by reptation theory and consistent with nonequilibrium thermodynamics, J. Non-Newtonian Fluid Mech. 101 (2001) 95-111.

[26] R.B. Bird, C.F. Curtiss, R.C. Armstrong, O. Hassager, Dynamics of Polymeric Liquids. Kinetic Theory, vol. 2, 2nd edition, Wiley, New York, 1987.

[27] X. Ye, R.G. Larson, C.J. Pattamaprom, T. Shridar, Extensional properties of monodisperse and bidisperse polystyrene solutions, J. Rheol. 47 (2003) $443-468$.

[28] J.D. Schieber, D.M. Nair, T. Kitkrailard, Comprehensive comparisons with nonlinear flow data of a consistently unconstrained Brownian slip-link model, J. Rheol. 51 (2007) 1111-1141.

[29] T. Schweizer, J. Meerveld, H.C. Ottinger, Nonlinear shear rheology of polystyrene melt with narrow molecular weight distribution-experiment and theory, J. Rheol. 48 (2004) 1345-1363. 